\title{
Entropy properties of deterministic control systems
}

\author{
Fritz Colonius
}

\begin{abstract}
The purpose of this tutorial paper is to explain and to survey a number of concepts and results for deterministic control systems centering on the problem to determine the "information" needed for performing control tasks. Entropy theory of dynamical systems provides a guideline in the sense that the developments for control systems are seen, sometimes in analogy and sometimes in contrast, to those for dynamical systems. A focus will be on the problem to keep a subset of the state space invariant. Furthermore, a number of open problems in this field will be elucidated.
\end{abstract}

\section{INTRODUCTION}

The purpose of this tutorial paper is to explain and to survey a number of concepts and results which are based on methods from the ergodic theory of dynamical systems. Probably [15] is the first paper to establish a connection between stabilization of control systems (based on quantized feedback) and ergodic theory (using invariant measures and the Perron-Frobenius operator). In the present paper, the focus will be on the problem to keep a subset of the state space (usually of full dimension) invariant. Furthermore, several open problems in this context will be elucidated.

Ergodic theory of dynamical systems, where the notion of entropy plays a decisive role, is a vast field and also its connections to control are diverse. Hence the present paper is not an effort to give a comprehensive survey. Instead, the narrative is concentrated on several main ideas and only scarce references to original contributions are included. In particular, we do not strive to present the most general settings and results. The emphasis is on situations where the concepts are intuitively appealing.

Entropy theory of dynamical systems goes back to Kolmogorov-Sinai (based on the pioneering work of Shannon) as well as Adler-Konheim-McAndrew, and concerns the problem to determine the total information generated by the system. In contrast, in entropy theory for control systems, the minimal information needed for the considered control task is of interest. This is closely related to minimal data rates which, in fact, provided the motivation in the seminal contribution [28]. For a survey on feedback under data rate constraints see [27].

In the literature, a number of different versions of invariance entropy for control systems has been introduced and a general agreement about notation has not yet been reached. Certainly, the list of open problems as well the choice of topics of this paper is strongly influenced by my own research interests and experience.

\footnotetext{
This work was supported by DFG grant Co 124/19-1

F. Colonius is with Institut für Mathematik, Universität Augsburg, 86159 Augsburg, Germany fritz.coloniusemath. uni-augsburg.de
}

The contents of this paper is as follows: In Section 2, main concepts and key results on metric (i.e., measuretheoretic) and topological entropy of dynamical systems are stated. Section 3 describes topological versions of invariance entropy for control systems and Section 4 discusses metric versions of invariance entropy. Extensions to other control problems like stabilization are briefly discussed.

\section{BACKGROUND ON ENTROPY IN THE THEORY OF DYNAMICAL SYSTEMS}

In this section, we sketch basic concepts and results for entropy in the theory of dynamical systems. The emphasis will be on general results for continuous flows on compact metric spaces, the differentiable theory will only briefly be touched upon.

As general references to the extensive literature on this field we mention the recent monograph [17], as well as the comprehensive monograph on dynamical systems [21, in particular, Chapters 3 and 4]. The text [32] provides a very careful introduction to the field. A detailed exposition of the fascinating development of entropy theory for dynamics is given in [20], while [33] gives a short and very readable exposition of the main results emphasizing the relation to Lyapunov exponents in the smooth case.

We will start with metric entropy, also called KolmogorovSinai entropy, which is based on measure theory. Let $\mu$ be a probability measure on a space $X$ endowed with a $\sigma$-algebra $\mathfrak{F}$. For every finite partition $\mathcal{P}=\left\{P_{1}, \ldots, P_{n}\right\}$ of $X$ into measurable sets the entropy is defined as

$$
H_{\mu}(\mathcal{P})=-\sum \mu\left(A_{i}\right) \log \mu\left(A_{i}\right) \quad=\int I_{\mu, \mathcal{P}}(x) \mu(d x),
$$

with the information function $I_{\mu, \mathcal{P}}(x):=-\log \mu\left(P_{x}\right)$ for the unique element $P_{x}$ of $\mathcal{P}$ with $x \in P_{x}$.

The sets in the partition $\mathcal{P}$ may be considered as the possible outcomes of an experiment. Then the entropy specifies the expected information gained from the outcomes of this experiment, or the amount of uncertainty removed, upon learning the $\mathcal{P}$-address of a randomly chosen point. Observe that the partition $\mathcal{P}$ generates a $\sigma$-algebra and the entropy is the information relative to this $\sigma$-algebra. Usually, the logarithm with base 2 is considered, however, for any base $a>0$ one has $\log _{2} z=\log _{a} z \cdot \log _{2} a$, hence this only differs by a constant. For continuous-time systems the natural logarithm is more appropriate.

Now let us look at a dynamical system (in discrete time) generated by a continuous map $T$ and its iterations on a compact metric space $X$. Here a natural choice of the relevant $\sigma$-algebra is the Borel $\sigma$-algebra, i.e., the smallest 
$\sigma$-algebra containing all open (or all closed) subsets of $X$. For two collections $\mathfrak{A}$ and $\mathfrak{B}$ of sets let the join be defined by

$$
\mathfrak{A} \vee \mathfrak{B}:=\{A \cap B \mid A \in \mathfrak{A} \text { and } B \in \mathfrak{B}\},
$$

and for a finite measurable partition $\mathcal{P}$ of $X$ and $j \in \mathbb{N}$ let

$$
T^{-j} \mathcal{P}:=\left\{T^{-j} P \mid P \in \mathcal{P}\right\}
$$

Then one finds that

$$
\mathcal{P}^{(n)}:=\bigvee_{j=0}^{n-1} T^{-j} \mathcal{P}=\mathcal{P} \vee T^{-1} \mathcal{P} \vee \ldots \vee T^{-(n-1)} \mathcal{P}
$$

again is a finite measurable decomposition of $X$. Here a natural choice for the probability measure $\mu$ is an invariant measure, that is,

$$
\mu\left(T^{-1} E\right)=\mu(E) \text { for all measurable } E \subset X .
$$

Then $\frac{1}{n} H_{\mu}\left(\mathcal{P}^{(n)}\right)$ is the per-iterate gain of information of the dynamical system $T$ with respect to $\mathcal{P}$. In the limit for $n \rightarrow \infty$, one obtains the entropy of $T$ with respect to the partition $\mathcal{P}$ as

$$
\left.h_{\mu}(T, \mathcal{P}):=\lim _{n \rightarrow \infty} \frac{1}{n} H_{\mu} \quad \mathcal{P}^{(n)}\right) .
$$

Finally, the metric entropy of $T$, i.e., the total information generated by the map $T$ with respect to $\mu$ is

$$
h_{\mu}(T):=\sup _{\mathcal{P}} h_{\mu}(T, \mathcal{P}),
$$

where the supremum is taken over all finite measurable partitions $\mathcal{P}$ of $X$. Here the idea is that coarse partitions $\mathcal{P}$ may overlook information that can be generated by the dynamical system. Hence one should look, in particular, at very fine partitions (it suffices to look at partitions with diameter tending to 0 [32, Theorem 8.3]). An important observation is that the limit in (1) exists. This relies on the subadditivity property

$$
\left.H_{\mu} \quad \mathcal{P}^{(m+n)}\right) \leq H_{\mu}\left(\mathcal{P}^{(m)}\right)+H_{\mu}\left(\mathcal{P}^{(n)}\right)
$$

for all $m, n \in \mathbb{N}$ and the following elementary subadditivity lemma.

Lemma 1: Let $\left(a_{n}\right)$ be a sequence in $\mathbb{R}$ with $a_{n+m} \leq$ $a_{n}+a_{m}$ for all $m, n \in \mathbb{N}$. Then $\lim _{n \rightarrow \infty} \frac{a_{n}}{n}$ exists.

The Shannon-McMillan-Breiman theorem (cf. [17, Theorem 3.3.1]) offers another interpretation of entropy. If $\mu$ is ergodic (i.e., the state space cannot per decomposed into two invariant subsets of positive measure), we define

$$
\mathcal{P}_{n}(x):=\left\{y \in X \mid P_{T^{i} x}=P_{T^{i} y} \text { for } 0 \leq i<n\right\} \in \mathcal{P}^{(n)} .
$$

This is the set of all $y$ following the same itinerary as $x$ up to time $n$. Then the following holds for all $x$ in a set of $\mu$-probability 1:

$$
\lim _{n \rightarrow \infty}-\frac{1}{n} \log \mu\left(\mathcal{P}_{n}(x)\right)=h_{\mu}(T, \mathcal{P}) .
$$

Thus, abbreviating $h=h_{\mu}(T, \mathcal{P})$ one has: Given any $\varepsilon>0$, there exists $N \in \mathbb{N}$ such that for all $n \geq N$ there is a set $X_{n} \subset X$ with $\mu\left(X_{n}\right)>1-\varepsilon$ such that
$X_{n}$ consists of approximately $e^{n(h \pm \varepsilon)}$ elements of $\mathcal{P}^{(n)}$ each having measure appr. $e^{-n(h \pm \varepsilon)}$. Viewing the elements of $\mathcal{P}^{(n)}$ in $X_{n}$ as representing "typical" $n$-itineraries, the Shannon-McMillan-Breiman theorem states that a system has entropy $h$ if the number of "typical" $n$-itineraries grows like $e^{n h}$. This gives another intuitive meaning for entropy.

In analogy to the metric entropy one can introduce a topological version (going back to [1]). Here measurable decompositions are replaced by finite open covers,

$$
\mathcal{A}:=\left\{A_{1}, \ldots, A_{n}\right\}
$$

with open sets $A_{i}$ such that $X=\bigcup_{i} A_{i}$. Then the set of itineraries of length $n$ is

$$
\mathcal{A}^{(n)}:=\bigvee_{j=0}^{n-1} T^{-j} \mathcal{A}=\left\{\bigcap_{j=0}^{n-1} T^{-j}\left(A_{j}\right) \mid A_{j} \in \mathcal{A}\right\},
$$

which again is an open cover. Denote the minimal cardinality (i.e., the minimal number of elements) of a subcover by $N\left(\mathcal{A}_{n}\right)$. Thus we remove all superfluous information. Then the topological entropy of $\mathcal{A}$ is given by

$$
\left.h(\mathcal{A}, T):=\lim _{n \rightarrow \infty} \frac{1}{n} \log N \quad \mathcal{A}^{(n)}\right)
$$

and the topological entropy of $T$ is

$$
h_{\text {top }}(T)=\sup _{\mathcal{A}} h(\mathcal{A}, T) .
$$

The limit in (3) exists by the same subadditivity property as in (2).

Basic properties of topological entropy are collected in the following proposition.

Proposition 2: Let $T: X \rightarrow X$ and $S: Y \rightarrow Y$ be continuous maps on compact metric spaces. Then the following holds:

(i) Power rule: $h_{\text {top }}\left(T^{k}\right)=k \cdot h_{\text {top }}(T)$ for all $k \in \mathbb{N}$.

(ii) Invariance under conjugacies: let $g: X \rightarrow Y$ be a homeomorphism with

$$
g \circ T=S \circ g .
$$

Then $h_{\text {top }}(T)=h_{\text {top }}(S)$.

Remark 1: The metric entropy also is invariant under appropriate conjugacies and satisfies the power rule. These results can be extended to dynamical systems in continuous time.

The relation between metric and topological entropy is clarified by the following variational principle (cf. [32, Theorem 8.6]).

Theorem 3: Let $T: X \rightarrow X$ be a continuous map on a compact metric space. Then

$$
h_{\text {top }}(T)=\sup \left\{h_{\mu}(T) \mid \mu \text { is } T \text {-invariant }\right\} .
$$

In general, there need not exist a measure $\mu$ with $h_{\mu}(T)=$ $h_{\text {top }}(T)$ (such a measure exists for expansive maps.)

There is another way to describe the topological entropy using explicitly the metric $d$ in $X$, going back to R. Bowen and E.I. Dinaburg. It counts the relevant objects in the state 
space and can be defined in a more general situation. For given $\varepsilon, \tau>0$ call $R \subset K$ an $(\varepsilon, \tau)$-spanning set if for every $x \in K$ there is $y \in R$ with

$$
d\left(T^{i} x, T^{i} y\right)<\varepsilon \text { for all } i=0, \ldots, \tau-1 .
$$

With $r(\tau, \varepsilon, K)$ denoting the minimal number of elements in such a set $R$ let

$$
h_{\text {span }}(\varepsilon, K, T):=\limsup _{n \rightarrow \infty} \frac{1}{n} \log r(n, \varepsilon, K)
$$

and

$$
h_{\text {span }}(K, T):=\lim _{\varepsilon \rightarrow 0} h_{\text {span }}(\varepsilon, K, T) .
$$

It turns out that for a compact metric space $X$ and $K=X$ this coincides with the topological entropy of $T$ as defined in (4),

$$
h_{\text {span }}(X, T)=h_{\text {top }}(T) .
$$

Remark 2: For the topological entropy it suffices to consider the restriction of the considered map $f$ to appropriate subsets of $X$ (cf. [31, Section 8.1]): The restriction of $T$ to the nonwandering set $\Omega$ which is invariant satisfies

$$
h_{\text {top }}(T)=h_{\text {top }}\left(T_{\mid \Omega}\right) .
$$

In fact, using the variational principle, Theorem 3, one finds that the topological entropy is already determined by the restriction of $T$ to the closure of the set of recurrent points.

Remark 3: Topological entropy on a uniformly hyperbolic set only depends on the periodic orbits inside this set, and a measure of maximal measure-theoretic entropy can be obtained as a limiting distribution of measures concentrated on periodic orbits.

The theory above may be generalized using ideas from statistical mechanics, leading to the so-called thermodynamic formalism. Here ideas by R. Bowen, Y. Sinai, and D. Ruelle were fundamental. If one analyzes the constructions for the entropy one discovers that they can be generalized in the following way:

For a function $\varphi$ in the space $C(X, \mathbb{R})$ of continuous functions from $X$ to $\mathbb{R}$ let

$$
\left(S_{n} \varphi\right)(x):=\sum_{i=0}^{n-1} \varphi\left(T^{i} x\right), x \in X
$$

and let

$$
Q_{n}(T, \varphi, \varepsilon):=\inf \left\{\sum_{x \in R} e^{\left(S_{n} \varphi\right)(x)} \mid R \text { is }(n, \varepsilon) \text {-spanning }\right\}
$$

and

$$
Q(T, \varphi, \varepsilon):=\limsup _{n \rightarrow \infty} \frac{1}{n} \log Q_{n}(T, \varphi, \varepsilon) .
$$

Furthermore, let

$$
P(T, \varphi):=\lim _{\varepsilon \rightarrow 0} Q(T, \varphi, \varepsilon)
$$

and define the topological pressure of $T$ as the map $P(T, \cdot)$ : $C(X, \mathbb{R}) \rightarrow \mathbb{R} \cup\{\infty\}$.

The topological pressure can also be defined by open covers. It is easily seen that for the map $\varphi \equiv 0$ one obtains
$P(T, \varphi)=h_{t o p}(T)$. The map $\varphi$ associates weights to the elements of $X$. A variational principle also holds for the pressure [32].

Theorem 4: Let $T: X \rightarrow X$ be a continuous map on a compact metric space. Then for every $\varphi \in C(X, \mathbb{R})$

$$
P(T, \varphi)=\sup h_{\mu}(T)+\int \varphi d \mu \mid \mu \text { is } T \text {-invariant } .
$$

Measures $\mu$ with $P(T, \varphi)=h_{\mu}(T)+\int \varphi d \mu$ are called equilibrium states (motivated from thermodynamics).

For differentiable systems, we only mention that entropy is connected with the (unstable) Lyapunov exponents which exist for almost all $x$ by the theorem of Oseledets. Here hyperbolicity conditions play an essential role. Furthermore, pressure is related to escape rates from neighborhoods of uniformly hyperbolic invariant sets; cf. [33, Section 3.2].

\section{TOPOLOGICAL INVARIANCE ENTROPY FOR CONTROL}

The basic question that underlies the following analysis is: What is the minimal information necessary to perform a given control task? This rather vague formulation can be made precise in different ways. Entropy theory of dynamical systems will provide a guideline for us in the sense that the concepts and results for control systems are seen, sometimes in analogy and sometimes in contrast, to the developments in dynamical systems theory.

Here it is helpful to look at control systems as dynamical systems. The simplest way of doing that is the following: Consider a discrete time system given by

$$
\begin{aligned}
x_{k+1} & =f\left(x_{k}, u_{k}\right), k \in \mathbb{N}_{0}=\{0,1, \ldots\} \\
u & =\left(u_{k}\right) \in \mathcal{U}:=\Omega^{\mathbb{N}_{0}},
\end{aligned}
$$

where $\Omega$ is the (nonvoid) control range and $f: M \times \Omega \rightarrow M$. To be definite, suppose that $M$ and $\Omega$ are metric spaces and $f: M \times \Omega \rightarrow M$ is continuous. Let $\varphi(k, x, u), k \in \mathbb{N}_{0}$, be the solution with initial condition $\varphi(0, x, u)=x$ and control $u$ and define the left shift

$$
\theta: \mathcal{U} \rightarrow \mathcal{U},\left(u_{k}\right) \mapsto\left(u_{k+1}\right) .
$$

(For a compact set $\Omega$ the space $\mathcal{U}$ becomes compact metrizable in the product topology and $\theta$ is continuous.) Then one obtains a dynamical system on $\mathcal{U} \times M$, called the control flow, by the iterations of the following skew product map $T: \mathcal{U} \times M \rightarrow \mathcal{U} \times M, T(u, x):=(\theta u, f(x, u))$ satisfying

$$
T^{k}(u, x)=\left(\theta^{k} u, \varphi(k, x, u)\right), k \in \mathbb{N}_{0} .
$$

The first component on the base space $\mathcal{U}$ does not depend on the second component, which has the cocycle property

$$
\varphi\left(l, \varphi(k, x, u), \theta^{k} u\right)=\varphi(l+k, x, u) \text { for } k, l \in \mathbb{N}_{0} .
$$

This is the point of view taken, e.g., in [11] for continuous time. This, definitely, is not the only (and probably not the most useful) view of control theory since, implicitly, it requires us to analyze the behavior of the system under arbitrary control functions. On the other hand, it certainly 
provides us with some intuition when we want to understand the relations to dynamical system theory.

Let us look at the problem to render a compact subset $Q$ of the state space invariant and suppose that $\Omega$ is compact. What is the information necessary to fulfill this task? For simplicity, we suppose that $Q$ satisfies the following weak invariance condition:

For all $x \in Q$ there is $\omega_{x} \in \Omega$ with $f\left(x, \omega_{x}\right) \in Q$.

In view of the definition of topological entropy for dynamical systems it is natural to look at open covers of $Q$ and the joins of their preimages. We will consider itineraries in $Q$. Hence, in order to take the invariance condition into account, we replace the open covers by invariant open covers defined as follows:

Definition 1: An invariant open cover is a triple $\mathcal{C}=$ $(\mathcal{A}, \tau, F)$, where $\mathcal{A}$ is an open cover of $Q, \tau \in \mathbb{N}$ and $F: \mathcal{A} \rightarrow \Omega^{\tau}$ with

$$
\varphi(i, A, F(A)) \subset Q \text { for } i=0, \ldots, \tau \text {. }
$$

This associates to every element $A$ in the open cover $\mathcal{A}$ a control function which, when applied to the elements in $A$, keeps the system in $Q$ up to time $\tau$. For a sequence $\alpha=\left(A_{0}, A_{1}, \ldots, A_{n-1}\right) \in \mathcal{A}^{n}$ define the control

$$
u(\alpha)=u(\alpha, \mathcal{C})=\left(F\left(A_{0}\right), F\left(A_{1}\right)(\tau+\cdot), \ldots\right) .
$$

It is obtained by applying the controls $F\left(A_{i}\right)$ on subsequent intervals of length $\tau$. Application of this control to an element $x$ of $A_{0}$ makes sense if after $\tau$ we are in $A_{1}$, etc. Then let

$$
\mathcal{C}_{n}(\alpha):=\left\{x \in Q \mid \varphi(i \tau, x, u(\alpha)) \in A_{i}, i=0, \ldots n-1\right\}
$$

and consider the family of all these itineraries

$$
\mathcal{C}^{(n)}:=\left\{\mathcal{C}_{n}(\alpha) \mid \alpha \in \mathcal{A}^{n}\right\} \text {. }
$$

Then $\mathcal{C}^{(n)}$ is an open cover of $Q$, and we denote the minimal cardinality of a subcover of $Q$ by $N\left(\mathcal{C}^{(n)} \mid Q\right)$. The invariance entropy of $\mathcal{C}$ is

$$
h_{\text {inv }}(\mathcal{C}, Q):=\lim _{n \rightarrow \infty} \frac{1}{n} \log N\left(\mathcal{C}^{(n)} \mid Q\right) .
$$

Using subadditivity one can show that the limit exists.

Definition 2: The topological feedback invariance entropy of $Q$ is

$$
h_{\text {inv }}(Q):=\inf _{\mathcal{C}} h_{\text {inv }}(\mathcal{C}, Q) .
$$

Here one may consider the (time-dependent) control functions $F(A):[0, \tau] \rightarrow \Omega$ as feedbacks, since they are applied to every element of $A$. This definition is essentially due to [28] who used (a strengthened version of) it to prove results about minimal data rates for stabilization about an equilibrium. In (9) an infimum is taken. This is a decisive difference to entropy for dynamical systems and is due to the fact that the minimal information necessary for invariance is of interest.

Another way is to consider the maps $T^{A}$ on $M$ given by $T^{A}:=\varphi(\tau, \cdot F(A))$. Then for sequences $\alpha \in \mathcal{A}^{n}$ one considers the associated sequences

$$
T^{A_{n-1}} \circ \ldots \circ T^{A_{0}} .
$$

Thus, instead of a single map $T$ as for dynamical systems, one considers for every invariant open cover $\mathcal{C}$ compositions of finitely many maps. This is similar to topological entropy for nonautonomous dynamical systems; cf. [25], [23].

The following alternative and more flexible construction (cf. [8]) is closer to the Bowen-Dinaburg version of topological entropy. It puts the emphasis on the control functions, instead of covers of the set $Q$ in the state space,

Let $K$ be a nonvoid subset of a compact set $Q \subset X$ and suppose that (7) holds. Given a time $\tau \in \mathbb{N}$ a set $\mathcal{S} \subset \mathcal{U}$ is called $(\tau, K, Q)$-spanning if for all $x \in K$ there is $u \in \mathcal{S}$ with

$$
\varphi(k, x, u) \in Q \text { for all } k \in\{0, \ldots, \tau\} .
$$

The minimal number of elements in such a set $\mathcal{S}$ is called $r_{\text {span }}(\tau, K, Q)$ (if no finite set $\mathcal{S}$ with this property exists, we set $\left.r_{\text {span }}(\tau, K, Q)=\infty\right)$.

Definition 3: With this notation, define the topological invariance entropy by

$$
h_{\text {inv }}(K, Q):=\limsup _{\tau \rightarrow \infty} \frac{1}{\tau} \log r_{\text {span }}(\tau, K, Q) .
$$

If $K=Q$, we just write $h_{i n v}(Q)$. For continuous time systems, the definition is completely analogous.

Remark 4: For $K=Q$ this definition is equivalent to feedback invariance entropy from Definition 2 based on invariant open covers, cf. [10] and [22, Theorem 2.3]).

In the case of linear control systems $\dot{x}=A x+B u$ with compact control range one obtains for the outer invariance entropy (which allows for small exits from $Q$ ) (cf. [22, Theorem 3.1]) the ubiquitous formula

$$
h_{\text {inv }, \text { out }}(Q)=\sum \max (\operatorname{Re} \lambda, 0)
$$

where summation is over all eigenvalues $\lambda$ of $A$ counted according to multiplicity. In this special case, the invariance entropy coincides with the topological entropy of the uncontrolled system $\dot{x}=A x$ (appropriately defined via spanning sets on the noncompact state space $\mathbb{R}^{d}$ ). In [14] this formula is generalized to linear control systems on Lie groups.

There are many results and estimates for topological invariance entropy (cf., in particular, Kawan [22]). We emphasize the following two results which illustrate the relations to dynamical systems theory. They are formulated for systems in continuous time and involve the following notions and facts from nonlinear control theory. Consider a control-affine system on $M=\mathbb{R}^{d}$ of the form

$$
\dot{x}=f_{0}(x)+\sum_{i=1}^{m} u_{i}(t) f_{i}(x)=f(x, u(t)), u \in \mathcal{U},
$$

where $f_{i}: \mathbb{R}^{d} \rightarrow \mathbb{R}^{d}$ are $C^{\infty}$ vector fields and the controls are taken in $\mathcal{U}=\left\{u: \mathbb{R} \rightarrow \mathbb{R}^{m} \mid u(t) \in \Omega\right.$ for all $t \in$ $\mathbb{R}$, locally integrable $\}$ with a nonvoid control range $\Omega \subset$ $\mathbb{R}^{m}$. For $x_{0} \in \mathbb{R}^{d}$ and $u \in \mathcal{U}$ the solution with $x(0)=x_{0}$ is again denoted by $\varphi\left(t, x_{0}, u\right), t \in \mathbb{R}$ (for simplicity, we assume global existence). For $W \subset \mathbb{R}^{d}$ the $W$-reachable set 
of $x \in W$ up to time $T>0$ is

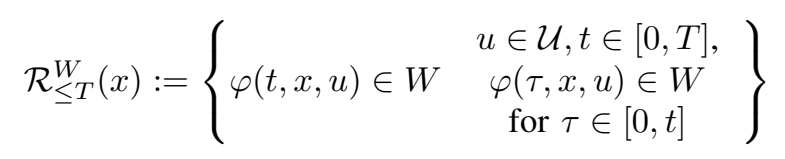

and

$$
\mathcal{R}^{W}(x):=\bigcup_{T>0} \mathcal{R}_{\leq T}^{W}(x)
$$

A $W$-control set $D$ is a maximal set (with respect to set inclusion) satisfying

$$
D \subset \operatorname{cl}_{W} \mathcal{R}^{W}(x) \text { for all } x \in D,
$$

where the closure is taken relative to $W$. A $W$-control set $C$ is called relatively invariant, if

$$
\mathrm{cl}_{W} C=\mathrm{cl}_{W} \mathcal{R}^{W}(x) \text { for all } x \in C .
$$

If $W=\mathbb{R}^{d}$ we omit the argument $W$.

Let $\mathcal{L}(f(\cdot, \omega) \mid \omega \in \Omega)$ be the Lie algebra generated by the vector fields of the control system, i.e., the smallest linear space of vector fields containing all $f(\cdot, \omega), \omega \in \Omega$, and closed under Lie brackets, thus $g, h \in \mathcal{L}(f(\cdot, \omega) \mid \omega \in \Omega)$ implies $[g, h] \in \mathcal{L}\{f(\cdot, \omega) \mid \omega \in \Omega\}$ with

$$
[g, h](x):=\frac{\partial h(x)}{\partial x} g(x)-\frac{\partial g(x)}{\partial x} h(x), x \in \mathbb{R}^{d} .
$$

The accessibility rank condition in a point $x \in \mathbb{R}^{d}$ requires

$$
\{g(x) \mid g \in \mathcal{L}(f(\cdot, \omega) \text { with } \omega \in \Omega)\}=\mathbb{R}^{d} .
$$

Condition (11) implies that $\mathcal{R}_{\leq T}(x)$ has nonvoid interior for every $T>0$.

For points $x, y \in \mathbb{R}^{d}$ and numbers $\varepsilon, \tau>0$ a controlled $(\varepsilon, \tau)$-chain from $x$ to $y$ is given by an integer $n \in \mathbb{N}$ and points $x_{0}, \ldots, x_{n} \in \mathbb{R}^{d}$, control functions $u_{0}, \ldots, u_{n-1} \in \mathcal{U}$ and times $t_{0}, \ldots, t_{n-1} \geq \tau$ such that $x_{0}=x, x_{n}=y$, and $\left\|\varphi\left(t_{i}, x_{i}, u_{i}\right)-x_{i+1}\right\|<\varepsilon$ for $i=0, \ldots, n-1$. A set $E \subset$ $\mathbb{R}^{d}$ is called a chain control set if it is a maximal set with the following properties:

(i) For all $x \in E$ there is $u \in \mathcal{U}$ with $\varphi(t, x, u) \in E$ for all $t \in \mathbb{R}$.

(ii) For all $x, y \in E$ and all $\varepsilon, \tau>0$ there exists an $(\varepsilon, \tau)$ chain from $x$ to $y$.

Thus chain control sets fulfill a weaker controllability property than control sets. They are modelled after chain transitive sets in the theory of dynamical systems.

The following result based on hyperbolicity is a special case of [13, Theorem 5.4].

Theorem 5: Let $E$ be a compact chain control set with nonvoid interior of system (5). Assume that

(i) the accessibility rank condition is satisfied on $\operatorname{int} E$ and for each $u \in \mathcal{U}$ there exists a unique $x(u) \in$ $E$ with $(u, x(u)) \in \mathcal{E}:=\{(u, x) \in \mathcal{U} \times$ $\mathbb{R}^{d} \mid \varphi(t, x, u) \in E$ for all $\left.t \in \mathbb{R}\right\}$.

(ii) the lift $\mathcal{E}$ of $E$ is uniformly hyperbolic, i.e., for each $(u, x) \in \mathcal{E}$ there exists a decomposition (of the tangent space in $x \in \mathbb{R}^{d}$ ) into stable and unstable subspaces, resp.,

$$
\mathbb{R}^{d}=E_{u, x}^{-} \oplus E_{u, x}^{+}
$$

such that the solution map $d_{x} \varphi(t, x, u)$ of the linearized system maps $E_{u, x}^{ \pm}$into $E_{u(t+\cdot), \varphi(t, x, u)}^{ \pm}$and there exist constants $0<c \leq 1$ and $\lambda>0$ such that for all $(u, x) \in \mathcal{E}$

$$
\begin{aligned}
& \left\|d_{x} \varphi(t, x, u)\right\| \leq c^{-1} e^{-\lambda t}\|v\| \text { for } v \in E_{u, x}^{-}, t \geq 0, \\
& \left\|d_{x} \varphi(t, x, u)\right\| \geq c e^{\lambda t}\|v\| \text { for } v \in E_{u, x}^{+}, t \geq 0 .
\end{aligned}
$$

Then $E$ is the closure of a control set $D$ and for every compact set $K \subset D$ of positive volume the topological invariance entropy is given by

$$
h_{i n v}(K, E)=\inf \limsup _{\tau \rightarrow \infty} \frac{1}{\tau} \log \sum_{i} \max \left(\lambda_{i}(u, x), 0\right) ;
$$

here the infimum is taken over all periodic points $(u, x) \in \mathcal{E}$ and the sum is taken over all Floquet exponents $\lambda_{i}(u, x)$ of the (periodic) differential equation obtained by linearizing along the periodic $(u, \varphi(\cdot, x, u))$.

Remark 5: (i) The proof of this result shows that there exists no strategy to make a hyperbolic chain control set invariant that cannot be beaten by the strategy of stabilizing the system at a periodic orbit inside the chain control set; cf. Remark 3 for a related result on reduction to periodic solutions of dynamical systems.

(ii) For small chain control sets that arise around hyperbolic equilibria the hyperbolicity condition as well as the condition that for each $u$ there exists a unique $x(u)$ with $(u, x(u)) \in \mathcal{E}$ are satisfied.

The next theorem from [12, Theorem 5.2] presents conditions which ensure that the topological invariance entropy is already determined on certain subsets of $Q$.

Theorem 6: Consider control system (5). Let $Q=$ $\operatorname{cl}(\operatorname{int} Q) \subset \mathbb{R}^{d}$ be compact with connected interior $W:=$ $\operatorname{int} Q$. Assume that the system satisfies accessibility rank condition (11) and that there is a closed set $Q_{0}$ contained in $W$ such that $Q_{0} \cap \operatorname{cl}_{W} \mathcal{O}^{W,+}(x) \neq \emptyset$ for every $x \in Q$. Let $K \subset Q$ be compact.

1) Then there are only finitely many relatively invariant $W$-control sets $C_{i}, i \in\{1, \ldots, n\}$ and for every $x \in K$ there is $C_{i} \subset \operatorname{cl}_{W} \mathcal{O}^{W,+}(x)$.

2) Suppose that for every relatively invariant $W$-control set $C_{i}$ the intersection of $K$ with $C_{i}$ contains a compact subset $K_{i}$ with nonvoid interior. Then the topological invariance entropy satisfies

$$
h_{\text {inv }}(K, Q)=\max _{i=1, \ldots, n} h_{i n v}\left(K_{i}, C_{i}\right) .
$$

Remark 6: This theorem gives conditions which ensure that the topological invariance entropy of $Q$ is already determined on the subset of $Q$ given by the union of the relatively invariant $W$-control sets; cf. Remark 2 for a related result in the theory of dynamical systems. Note also that the lifts to $\mathcal{U} \times Q$ of the control sets are the maximal topologically transitive sets of the control flow, while the lifts $\mathcal{E}$ of the chain control sets $E$ are the connected subsets of the chain recurrent set, cf. [11, Chapter 4].

The following example of a chemical reactor illustrates Theorems 5 and 6. It concerns control of a continuous stirred 
tank reactor (CSTR) with Arrhenius' dynamics, cf., e.g., [30] or [18]. Consider

$$
\begin{aligned}
& \dot{x}_{1}=-x_{1}-a\left(x_{1}-x_{c}\right)+B \alpha\left(1-x_{2}\right) e^{x_{1}}+u(t)\left(x_{c}-x_{1}\right) \\
& \dot{x}_{2}=-x_{2}+\alpha\left(1-x_{2}\right) e^{x_{1}} .
\end{aligned}
$$

Here $x_{1}$ is the (dimensionless) temperature; $x_{2}$ is the product concentration; and $a, \alpha, B$, and $x_{c}$ are positive constants. The parameter $x_{c}$ is the coolant temperature, and hence the control affects the heat transfer coefficient. Specifically, we take

$$
\begin{aligned}
a & =0.15, \alpha=0.05, B=7.0, x_{c}=1.0, \\
\Omega & =[-\rho, \rho], 0<\rho \leq 0.15 .
\end{aligned}
$$

Because of the physical constraints, we consider the system in the set $[0, \infty) \times[0,1] \subset \mathbb{R}^{2}$. Let $y_{i}=\alpha e^{z_{i}} /(1+$ $\left.\alpha z_{i}\right), \quad i=0,1,2$, where $z_{1}<z_{0}<z_{2}$ are the zeros of the transcendental equation

$$
-z-(a+\omega)\left(z-x_{c}\right)+B \alpha \quad 1-\frac{\alpha e^{z}}{1+\alpha e^{z}} \quad e^{z}=0 .
$$

For every $\omega \in \Omega$ there are two stable equilibria given by $x_{1}=\left(z_{1}, y_{1}\right)$ and $x_{2}=\left(z_{2}, y_{2}\right)$ and a hyperbolic equilibrium $x_{0}=\left(z_{0}, y_{0}\right)$, i.e., the linearization about $x_{0}$ has one negative and one positive eigenvalue.

The system satisfies accessibility rank condition (11) at every point of the forward invariant set $(0, \infty) \times(0,1)$. The numerical computations indicate that for the parameter values above the set $M=[0,7] \times[0,1]$ contains exactly three control sets $C_{1}, C_{2}$, and $D$ containing the fixed points $x_{i}(\omega), i=1,2,0$, for $\omega \in$ int $\Omega=(-\rho, \rho)$ in their interiors. The control sets $C_{1}$ and $C_{2}$ are invariant; the control set $D$ is variant, see Figure 1 for the situation with $\rho=0.15$.

An interesting feature of this system is that the stable fixed point $x_{2}$ with the highest product concentration cannot be realized for technical reasons, hence the system should be kept near the hyperbolic equilibrium $x_{0}$. Thus it is of interest to determine the invariance entropy for initial points which can be steered into the control set $D$.

In [11, Section 9.1] the following results are shown (partially based on numerical evidence) for the controllability structure of this system. Denote by $M^{+}(\omega)$ and $M^{-}(\omega)$ the stable and the unstable manifolds, respectively, in $M$ of the hyperbolic fixed points $x_{0}(\omega), \omega \in[-0.15,0.15]$. Then the control set $D$ is given by

$$
\operatorname{int} D=\operatorname{int} \bigcup_{\omega_{1}, \omega_{2} \in \Omega}\left(M^{+}\left(\omega_{1}\right) \cap M^{-}\left(\omega_{2}\right)\right),
$$

and the domain of attraction of $D$ defined as $\mathbf{A}(D):=\{y \in$ $\left.M \mid \operatorname{clO}^{M,+}(y) \cap D \neq \varnothing\right\}$ satisfies

$$
\mathbf{A}(D)=\operatorname{int} \bigcup_{\omega \in \Omega} W^{+}(\omega) .
$$

The boundary of $\mathbf{A}(D)$ consists of the stable manifolds corresponding to $\omega_{1}=-\rho$ and $\omega_{2}=\rho$.

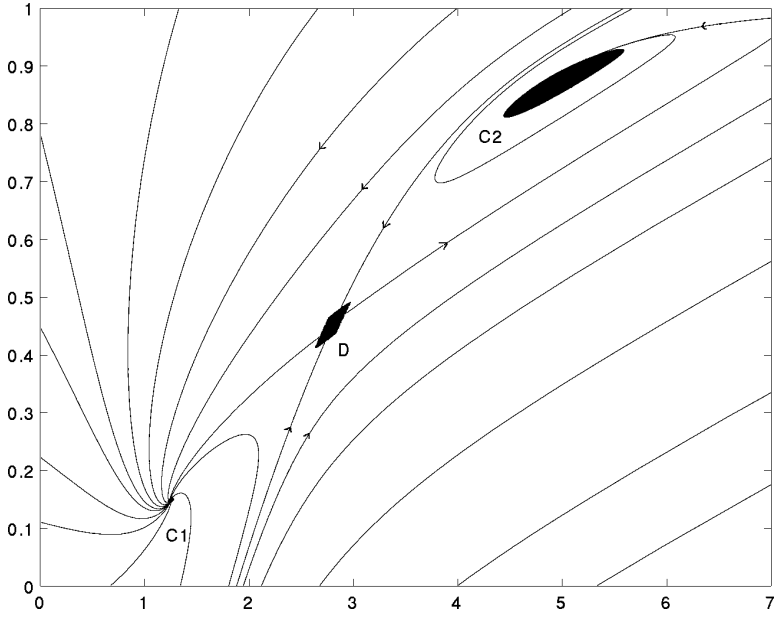

Fig. 1. Phase portrait of the continuous flow stirred tank reactor and control sets

Let $\rho>0$ be small enough. Then Theorem 5 implies that for every compact set $K \subset D$ with nonvoid interior the topological invariance entropy is given by

$$
\begin{aligned}
& h_{\text {inv }}(K, \mathrm{cl} D) \\
& =\inf \limsup _{\tau \rightarrow \infty} \frac{1}{\tau} \log \sum_{i} \max \left(\lambda_{i}(u, x), 0\right) ;
\end{aligned}
$$

here, with $E=\operatorname{cl} D$ the infimum is taken over all periodic $(u, x) \in \mathcal{E}$ and the sum is taken over all Floquet exponents of the (periodic) differential equation obtained by linearizing along the periodic $(u, x)$.

In fact, uniform hyperbolicity of the control system restricted to the lift $\mathcal{E}$ can be verified, since the equilibrium of the uncontrolled system is hyperbolic. Then roughness of the Sacker-Sell spectrum (or the Morse spectrum, cf. e.g., Colonius and Kliemann [11, Corollary 5.3.11]) implies that uniform hyperbolicity also holds for $\rho>0$ small enough.

Furthermore, for $\rho \rightarrow 0$, one can show that the spectrum converges (in the Hausdorff metric) to the set consisting of the eigenvalues of the hyperbolic equilibrium $x_{0}=\left(z_{0}, y_{0}\right)$ corresponding to the control $u=0$.

Theorem 6 can be applied with $W:=\mathbf{A}(D)$. The set $W$ is open, relatively compact and connected. The control set $D$ is a relatively invariant $W$-control set. Hence it follows that for every compact set $K \subset \mathbf{A}(D)$ such that $K$ intersects $\operatorname{int} D$ in a set with nonvoid interior the invariance entropy satisfies $h_{i n v}(K, \mathbf{A}(D))=h_{i n v}(K, D)$.

Further work: The encyclopedia entry [24] gives condensed information on topological invariance entropy. The growth rate of the number of open-loop control functions as a measure for the information needed to achieve invariance can also be used for other control tasks: In [3] this is done for exponential stabilization; in [7] and in [4] for controlled invariant subspaces. The contribution [6] discusses generalizations of topological invariance entropy to semigroup actions.

Instead of entropy for invariance of the state one may also analyze invariance properties of outputs. This is done for 
feedback invariance entropy in [19] and for outer invariance entropy in [9]. [22, Chapter 6] shows that appropriately defined escape rates are closely related to topological invariance entropy.

\section{METRIC INVARIANCE ENTROPY FOR CONTROL}

In this section we present measure-theoretic versions of invariance entropy. Recall from (6) that control system (5) can be described by the map $T(u, x)=\left(\theta u, f\left(x, u_{0}\right)\right)$ on the space $\mathcal{U} \times M$. The topological invariance entropy looks at the preimages under $T$ of invariant open covers replacing open covers. In analogy to metric entropy of dynamical systems we will construct metric invariance entropy by considering invariant partitions which will replace partitions.

An important point is the choice of an appropriate probability measure with respect to which entropy will be defined. Since we want to make $Q$ invariant, one might, for an appropriately defined feedback, consider an invariant measure on $Q$ (this is an approach followed by [15] and [26] who analyze a corresponding Perron-Frobenius operator; it should be noted that analysis of the Perron-Frobenius operator and of its adjoint, the Koopman operator, are very active fields of research in applied dynamical systems.) We will follow a different path which is more in line with the notion of topological invariance entropy. We look at conditionally invariant probability measures on the product space $\mathcal{U} \times M$ which describe the portion of the mass that is not allowed to return from outside of $Q$ into $Q$. The concept is most easily understood in the special case of quasi-stationary measures.

Fix a probability measure $\nu_{0}$ on the Borel $\sigma$-algebra $\mathcal{B}(\Omega)$ of the control range $\Omega$. Then $x_{k+1}=f\left(x_{k}, u_{k}\right)$ can be considered as iterations of a random map $f$.

Let $p(x, A):=\nu_{0}\{\omega \in \Omega \mid f(x, \omega) \in A\}, x \in M, A \subset$ $M$, be the associated Markov transition probabilities. Again, fix a compact subset $Q$ of $M$. A quasi-stationary measure with respect to $Q$ for the random map $f$ is a probability measure $\eta$ on $\mathcal{B}(M)$ such that $0<\rho:=\int_{Q} p(x, Q) d \eta \leq 1$ and

$$
\rho \cdot \eta(A)=\int_{Q} p(x, A) d \eta \text { for all } A \in \mathcal{B}(Q) .
$$

Thus the probability to go from $Q$ into $A$ is equal to a constant proportion of the probability of $A$. Putting $A=Q$, one sees that the support of $\eta$ is contained in $Q$. Observe that $1-\int_{Q} p(x, Q) d \eta$ is the average probability to exit in one step from $Q$. The measure $\eta$ is stationary, if and only if $\int_{Q} p(x, Q) d \eta=1$.

A closer look at quasi-stationary measures shows that they define probability measures on the product space $\mathcal{U} \times M$ : Define $\nu:=\nu_{0}^{\mathbb{N}}$ as the product measure on $\mathcal{U}=\Omega^{\mathbb{N}}$. Then, by [5, Proposition 2.8],

$$
\mu:=\nu \times \eta=\nu_{0}^{\mathbb{N}} \times \eta
$$

is a probability measure with

$$
\rho \cdot \mu(B)=\mu\left(T^{-1} B \cap(\mathcal{U} \times Q)\right)
$$

for all $B \in \mathcal{B}(\mathcal{U} \times M)$ where

$$
\rho:=\mu\left(T^{-1}(\mathcal{U} \times Q) \cap(\mathcal{U} \times Q)\right)=\int_{Q} p(x, Q) d \eta .
$$

The measure $\mu$ takes into account that the system cannot return from the hole $\mathcal{U} \times(M \backslash Q)$ into $\mathcal{U} \times Q$.

In fact, the special construction of $\mu$ in (13) is not relevant for the following. Instead one can take any probability measure $\mu$ with property (14).

Definition 4: A conditionally invariant measure $\mu$ for the map $T$ defined in (6) with respect to a compact subset $Q$ of $M$ is a probability measure on the Borel $\sigma$-algebra of $\mathcal{U} \times M$ such that

$$
\rho \cdot \mu(B)=\mu\left(T^{-1} B \cap(\mathcal{U} \times Q)\right)
$$

for all $B \in \mathcal{B}(\mathcal{U} \times M)$ and $0<\rho:=\mu\left(T^{-1}(\mathcal{U} \times Q) \cap(\mathcal{U} \times\right.$ $Q)) \leq 1$

Quasi-stationary measures are a classical object in stochastics; cf. the recent monograph [2]. Conditionally invariant measures for dynamical systems go back to [29], a survey is given in [16].

We denote by $T_{Q}:=T_{\mid \mathcal{U} \times Q}$ the restriction of $T$. The support of a conditionally invariant measure as above is contained in

$$
\left\{(u, x) \in \mathcal{U} \times Q \forall k \in \mathbb{N}: T_{Q}^{-k}(u, x) \neq \emptyset\right\}
$$

and for $k \in \mathbb{N}$ the measure $\mu$ is also conditionally invariant for $T_{Q}^{k}$ with constant $\rho^{k}$.

Again we suppose that the compact set $Q$ satisfies the invariance condition (7). Since $\mu$ lives in $\mathcal{U} \times Q$ we construct certain partitions for $\mathcal{U} \times Q$ whose entropy with respect to $\mu$ will be used to define the metric invariance entropy. While this seems fairly straightforward for the component in $Q$, more work will be needed for the $\mathcal{U}$-component. Again, we will consider itineraries in $Q$. Hence we start with the following definition referring to forward invariance (taken from [22, Definition 2.8])

Definition 5: An invariant partition of $Q$ is a triple $\mathcal{C}=$ $(\mathcal{P}, \tau, F)$ where $\mathcal{P}$ is a finite partition of $Q$ into Borel measurable sets, $\tau \in \mathbb{N}$, and $F: \mathcal{P} \rightarrow \Omega^{\tau}$ is a map assigning to each set $P$ in $\mathcal{P}$ a control function such that $\varphi(k, P, F(P)) \subset Q$ for all $k \in\{0,1, \ldots, \tau\}$.

In terms of the skew product map $T$ on $\mathcal{U} \times M$ (cf. (6)), the condition for an invariant partition $(\mathcal{P}, \tau, F)$ means that for all $P \in \mathcal{P}$ the map $T$ satisfies

$$
\begin{aligned}
T^{k}(F(P), P) & =\left(\theta^{k}(F(P)), \varphi(k, P, F(P))\right) \\
& \subset \mathcal{U} \times Q \text { for } k=0, \ldots, \tau .
\end{aligned}
$$

One easily sees that for an invariant open cover $(\mathcal{A}, \tau, G)$ there is also an invariant partition $(\mathcal{P}, \tau, F)$ with $\# \mathcal{P} \leq \# \mathcal{A}$. In the quasi-stationary case, where the conditionally invariant measure is of the form $\mu=\nu_{0}^{\mathbb{N}} \times \eta$, we may relax the condition on the considered partitions $\mathcal{P}$ by neglecting sets of $\eta$-measure zero in $Q$.

For a sequence $\alpha=\left(P_{i}\right) \in \mathcal{P}^{n}$ define a corresponding control $u(\alpha, \mathcal{C})$ as in (8). Such a sequence $\alpha \in \mathcal{P}^{n}$ of 
partition elements is called a $\mathcal{C}$-itinerary, if there is $x \in Q$ with $\varphi(i \tau, x, u(\alpha, \mathcal{C})) \in P_{i}$ for all $i$. By the definition of an invariant partition, one has $\varphi\left(k, P_{i}, F\left(P_{i}\right)\right) \subset Q$ for all $k \in\{0, \ldots, \tau\}$ and all $i$, and hence it follows that $\varphi(k, x, u(\alpha, \mathcal{C})) \in Q$ for all $k=0, \ldots,(n-1) \tau$.

Next we define the feedback invariance $\mu$-entropy. Fix an invariant partition $\mathcal{C}=(\mathcal{P}, \tau, F)$. For every $\mathcal{C}$-itinerary $\alpha=$ $\left(P_{0}, \ldots, P_{n-1}\right) \in \mathcal{P}^{n}$ define

$$
\begin{aligned}
& \mathcal{C}_{n}(\alpha) \\
& :=\left\{\begin{array}{cc}
\exists \mathcal{D}=(\mathcal{P}, \tau, G): u=u(\alpha, \mathcal{D}), \\
(u, x) \in \mathcal{U} \times Q & \forall i=0, \ldots, n-1: \\
\varphi(i \tau, x, u) \in P_{i}
\end{array}\right\} .
\end{aligned}
$$

Clearly, there is a pair $(u(\alpha, \mathcal{C}), x)$ in $\mathcal{C}_{n}(\alpha)$. If the sequence $\alpha$ is not a $\mathcal{C}$-itinerary, we let $\mathcal{C}_{n}(\alpha):=\emptyset$. The set $\mathcal{C}_{n}(\alpha)$ consists of all pairs $(u(\alpha, \mathcal{D}), x)$ with the same itinerary under an invariant partition $\mathcal{D}$ (with a feedback $G$ on the same partition $\mathcal{P}$ and $\tau$ as for $\mathcal{C}$ ).

The sets $\mathcal{C}_{n}(\alpha), \alpha \in \mathcal{P}^{n}$, together with the complement $Z_{n}^{\alpha}$ in $\mathcal{U} \times Q$ of its union form a measurable partition $\mathcal{C}^{(n)}$ of $\mathcal{U} \times Q$ and its entropy is given by

$$
\begin{aligned}
H_{\mu}\left(\mathcal{C}^{(n)}\right)=- & \sum_{\alpha \in \mathcal{P}^{n}} \mu\left(\mathcal{C}_{n}(\alpha)\right) \log \mu\left(\mathcal{C}_{n}(\alpha)\right) \\
& -\mu\left(Z_{n}^{\alpha}\right) \log \mu\left(Z_{n}^{\alpha}\right) .
\end{aligned}
$$

This entropy describes the amount of information with respect to $\mu$ needed to describe the sets $\mathcal{C}_{n}(\alpha)$ as subsets of $\mathcal{U} \times Q$. Note also that sets of vanishing $\mu$-measure do not play a role here (as usual, we let $-x \log x:=0$ for $x=0$ and 1).

Next we let

$$
h_{\mu}^{f b}(\mathcal{C}):=\lim _{n \rightarrow \infty} \frac{1}{n \tau} H_{\mu}\left(\mathcal{C}^{(n)}\right) .
$$

One can prove that the limit exists. Finally, we define the metric feedback invariance entropy in the following way.

Definition 6: For the skew product map $T$ from (6) determining control system (5) the feedback invariance $\mu$-entropy of $Q$ with respect to a conditionally invariant measure $\mu$ is defined by

$$
h_{\mu}^{f b}(Q, T):=\inf _{\mathcal{C}} h_{\mu}^{f b}(\mathcal{C})
$$

where the infimum is taken over all invariant partitions $\mathcal{C}$ of $Q$. If no invariant partition exists, we define $h_{\mu}^{f b}(Q, T):=$ $\infty$.

Again, we emphasize that here an infimum is taken, in contrast to the usual metric entropy of dynamical systems, since we are interested in the minimal information needed to make $Q$ forward invariant.

Instead of restricting the analysis to invariant partitions $\mathcal{D}$ one may also consider arbitrary controls which allow us to follow the same itineraries as $\mathcal{C}$. This leads to the following construction. For a $\mathcal{C}$-itinerary $\alpha=\left(P_{0}, \ldots, P_{n-1}\right) \in \mathcal{P}^{n}$ and $n \geq 1$ define

$$
\begin{aligned}
& \mathcal{C}_{n}(\alpha) \\
& :=\left\{\begin{array}{cc}
\forall i=0, \ldots, n-1 \forall k=0, \ldots, \\
(u, x) \in \mathcal{U} \times Q \quad(n-1) \tau: \varphi(k, x, u) \in Q \\
\text { and } \varphi(i \tau, x, u) \in P_{i}
\end{array}\right\} .
\end{aligned}
$$

By definition, there is a pair $(u(\alpha, \mathcal{C}), x)$ in $\mathcal{C}_{n}(\alpha)$. If $\alpha \in \mathcal{P}^{n}$ is not a $\mathcal{C}$-itinerary, we let $\mathcal{C}_{n}(\alpha):=\emptyset$. The set $\mathcal{C}_{n}(\alpha)$ consists of all pairs $(u, x)$ with the same itinerary as for $\mathcal{C}$. Note that here the control $u$ should not be interpreted as a feedback, but as an open loop control.

The sets $\mathcal{C}_{n}(\alpha), \alpha \in \mathcal{P}^{n}$, together with the complement $Z_{n}^{\alpha}$ in $\mathcal{U} \times Q$ of its union form a measurable partition $\mathcal{C}^{(n)}$ of $\mathcal{U} \times Q$ and its entropy is given by

$$
\begin{aligned}
H_{\mu}\left(\mathcal{C}^{(n)}\right)=- & \sum_{\alpha \in \mathcal{P}^{n}} \mu\left(\mathcal{C}_{n}(\alpha)\right) \log \mu\left(\mathcal{C}_{n}(\alpha)\right) \\
& -\mu\left(Z_{n}^{\alpha}\right) \log \mu\left(Z_{n}^{\alpha}\right) .
\end{aligned}
$$

This entropy describes the amount of information with respect to $\mu$ needed to describe the sets $\mathcal{C}_{n}(\alpha)$ as subsets of $\mathcal{U} \times Q$.

Next we let

$$
h_{\mu}^{c o}(\mathcal{C}):=\lim _{n \rightarrow \infty} \frac{1}{n \tau} H_{\mu}\left(\mathcal{C}^{(n)}\right) .
$$

One can prove that the limit exists. Finally, we define the metric controlled invariance entropy in the following way.

Definition 7: For the skew product map $T$ from (6) determining control system (5) the controlled invariance $\mu$-entropy of $Q$ with respect to a conditionally invariant measure $\mu$ is defined by

$$
h_{\mu}^{c o}(Q, T):=\inf _{\mathcal{C}} h_{\mu}^{c o}(\mathcal{C})
$$

If no invariant partition exists, we define $h_{\mu}^{c o}(Q, T):=\infty$.

Both versions of metric invariance entropy satisfy the standard properties of entropy, invariance under appropriately defined conjugacies and the power rule. The proof of the latter property requires the existence of the limit in (15) and (16), respectively.

The following relations between the invariance entropies hold [5, Theorem 4.4].

Theorem 7: Let $Q$ be a compact subset of the state space of control system (5) satisfying condition (7). Consider for the associated skew product map $T$ from (6) the topological invariance entropy as well as the feedback invariance $\mu$ entropy and the controlled invariance $\mu$-entropy with respect to a conditionally invariant measure $\mu$. Then the following inequalities hold:

$$
h_{\mu}^{f b}(Q, T) \leq h_{\mu}^{c o}(Q, T) \leq h_{i n v}(Q, T) .
$$

\section{CONCLUSIONS AND FURTHER WORK}

Entropy for control differs from entropy for dynamical systems by the fact, that for control the minimal required information is of interest instead of the entropy generated by the system. Here are some open questions and conjectures. 
(i) Does the supremum over all metric invariance entropies coincide with the topological invariance entropy, i.e., is a variational principle valid as for entropy of dynamical systems?

(ii) If the answer is yes, does a similar variational principle also hold for pressure (cf. Theorem 4)?

(iii) Is $h_{\mu}^{f b}(Q, T)=h_{\mu}^{c o}(Q, T)$ for every conditionally invariant measure $\mu$ ? By Remark 4 this is valid in the topological case.

(iv) Is there an analogue to the Shannon-McMillanBreiman theorem for metric invariance entropy (for appropriately defined "ergodic" conditionally invariant measures $\mu)$ ?

More specifically, define for an invariant partition $\mathcal{C}=$ $(\mathcal{P}, \tau, F)$ and a $\mathcal{C}$-itinerary $\alpha=\left(P_{0}, \ldots, P_{n-1}\right) \in \mathcal{P}^{n}$ and $(u(\alpha), x) \in \mathcal{U} \times Q$

$$
\begin{aligned}
& \mathcal{C}_{n}(u(\alpha), x) \\
& :=\left\{\begin{array}{cc}
(v, y) \in \mathcal{U} \times Q & \exists \mathcal{D}=(\mathcal{P}, \tau, G): v=v(\alpha, \mathcal{D}), \\
& \forall i=0, \ldots, n-1: \\
& \varphi(i \tau, x, u(\alpha)), \varphi(i \tau, y, v) \in P_{i}
\end{array}\right\} .
\end{aligned}
$$

This is the set of all $(v, y)$ following the same itinerary as $(u(\alpha), x)$ up to time $n$. What is the relation between

$$
\begin{gathered}
\lim _{n \rightarrow \infty}-\frac{1}{n} \log \mu\left(\mathcal{C}_{n}(u(\alpha), x)\right) \text { and } h_{\mu}(T, \mathcal{C}) ? \\
\text { REFERENCES }
\end{gathered}
$$

\section{REFERENCES}

[1] R.Adler, A.Konheim, and M.McAndrew, Topological entropy, Trans. Amer. Math. Soc., vol. 114, 1965, pp 61-85

[2] P.Collett, S.Martinez, and J.San Martin, Quasi-Stationary Distributions: Markov Chains, Diffusions and Dynamical Systems, SpringerVerlag, 2013

[3] F.Colonius, Minimal bit rates and entropy for stabilization, SIAM $J$. Control Optim., vol. 50, 2012, pp 2088-3010

[4] F.Colonius, Subspace entropy and controlled invariant subspaces, In Mathematical System Theory - Festschrift in Honor of Uwe Helmke on the Occasion of his Sixtieth Birthday, Createspace, 2013

[5] F.Colonius, Metric invariance entropy and conditionally invariant measures, submitted, 2015.

[6] F.Colonius, R.Fukuoka, and A.J. Santana, Invariance entropy for topological semigroup actions, Proc. Amer. Math. Soc., vol. 141, 2013, pp 4411-4423

[7] F.Colonius and U.Helmke, Entropy of controlled invariant subspaces, Zeitschrift fr Angewandte Mathematik und Mechanik, vol. 94, 2014, pp 331-344

[8] F.Colonius and C.Kawan, Invariance entropy for control systems, SIAM J. Control Optim., vol. 48, 2009, pp 1701-1721

[9] F.Colonius and C.Kawan, Invariance entropy for outputs, Mathematics of Control, Signals, and Systems, vol. 22(3), 2011, pp 203-227

[10] F.Colonius, C.Kawan, and G.Nair, A note on topological feedback entropy and invariance entropy, Systems and Control Letters, vol. 62, pp 377-381

[11] F.Colonius and W.Kliemann, The Dynamics of Control, Birkhäuser, Boston, 2000.

[12] F.Colonius and R.Lettau, Relative controllability properties, IMA Journal of Mathematical Control and Information, 2015, doi:10.1093/imamci/dnv004

[13] A.da Silva and C.Kawan, Invariance entropy of hyperbolic control sets, Discrete and Continuous Dynamical Systems A, vol. 36, 2016, pp $97-136$

[14] A.da Silva, Outer invariance entropy for linear systems on Lie groups, SIAM J. Control Optim., vol. 52, 2009, pp 1701-1721

[15] D.F. Delchamps, Stabilizing a linear system with quantized state feedback, IEEE Trans. Aut. Control, vol. 35, 1990, pp 916-924

[16] M.F. Demers and L.S. Young, Escape rates and conditionally invariant measures, Nonlinearity, vol. 19, 2006, pp 377-397

[17] T.Downarowicz, Entropy in Dynamical Systems, Cambridge University Press, 2011
[18] M.Golubitsky and D.G. Schaeffer, Singularities and Groups in Bifurcation Theory, Springer-Verlag, 1985

[19] R.Hagihara and G.Nair, Two extensions of topological feedback entropy, Math. Control Signals Syst., vol. 25, 2013, pp 473-490

[20] A.Katok, Fifty years of entropy in dynamics: 1958-2007, J. of Modern Dynamics, vol. 1(4), 2007, pp 545-596

[21] A.Katok and B.Hasselblatt, Introduction to the Modern Theory of Dynamical Systems, Cambridge University Press, 1995

[22] C.Kawan, Invariance Entropy for Deterministic Control Systems. An Introduction, volume 2089 of Lecture Notes in Mathematics, SpringerVerlag, 2013

[23] C.Kawan, Metric entropy of nonautonomous dynamical systems, Nonautonomous and Stochastic Dynamical Systems, vol. 1, 2013, pp 26-52

[24] C.Kawan, Data-rate of nonlinear control systems and feedback entropy, In Tariq Samad and John Baillieul, editors, Encyclopedia of Systems and Control, Springer-Verlag, 2014

[25] S.Kolyada and L.Snoha, Topological entropy for nonautonomous dynamical systems, Random and Computational Dynamics, vol. 4, 1996, pp 205-233

[26] P.G. Mehta, U.Vaidya, and A.Banaszuk, Markov chains, entropy, and fundamental limitations in nonlinear stabilization, IEEE Trans. Aut. Control, vol. 53(3), 2008, pp 784-791.

[27] G.N. Nair, F.Fagnani, S.Zampieri, and R.J. Evans, Feedback control under data rate constraints: an overview, Proceedings of the IEEE, vol. 95(1), 2007, pp 108-137

[28] G.N. Nair, R.J. Evans, I.M.Y. Mareels, and W.Moran, Topological feedback entropy and nonlinear stabilization, IEEE Trans. Aut. Control, vol. 49, 2004, pp 1585-1597

[29] G.Pianigiani and J.A. Yorke, Expanding maps on sets which are almost invariant: Decay and chaos, Trans. Amer. Math. Soc., vol. 252, 1979, pp 351-366

[30] A.B. Poore, A model equation arising from chemical reactor theory, Arch. Rational Mech. Anal., vol. 52, 1974, pp 358-388

[31] C.Robinson, Dynamical Systems. Stability, Symbolic Dynamics, and Chaos, CRC Press, 1999, Second edition

[32] P.Walters, An Introduction to Ergodic Theory, Springer-Verlag, 1982.

[33] L.-S. Young, Mathematical theory of Lyapunov exponents, J. Phys. A: Math.Theor., vol. 46 (254001): 17 pp, 2013 